Pacific Journal of Mathematic 


\title{
CONTINUOUS LINEAR IMAGES OF PSEUDO-COMPLETE LINEAR TOPOLOGICAL SPACES
}

\author{
AARON R. TODD
}

The various properties of topological spaces in the classical Baire category theorems which imply the Baire property also imply the stronger property of pseudo-completeness. In contrast to some of these properties and to the Baire property, J. C. Oxtoby has shown that pseudo-completeness is productive. The following main result places pseudo-completeness in the context of linear topological spaces: Let $E$ and $F$ be linear topological spaces and $g$ be a continuous linear mapping of $E$ into $F$. If $E$ is pseudo-complete, $g$ is almost open, and the completion of $g[E]$ has a continuous metric, then $g[E]$ is complete. The proof of this result uses the difference theorem, but not an open mapping theorem. The hypotheses lead to a discussion of conditions for a linear mapping to be almost open and for a linear topological space to have a continuous metric.

An example shows that, although a translation invariant continuous metric on a linear topological space $E$ extends to a translation invariant continuous pseudo-metric on the completion of $E$, this extension need not be a metric, even if it induces a normed topology on $E$. Othes examples show that a pseudo-complete linear topological space need not be complete in its natural uniformity, and that the almost open condition of the main result may not be omitted and is not implied by the combination of the other conditions and the conclusion.

Pseudo-complete topological spaces, introduced by Oxtoby [8], successfully bind together the classical Baire category theorems. They have been the subject of recent work; Aarts and Lutzer [1], for example, have shown that a metrizable space is pseudo-complete if and only if it has a dense subspace which is topologically complete. In the context of linear topological spaces, Todd [13] shows that, under certain conditions, a linear topological space which is pseudocomplete is complete in the natural uniformity. Theorem 3.1 extends this result for the image of a pseudo-complete linear topological space under a continuous linear almost open mapping; in contrast, Example 5.5 gives pseudo-complete locally convex linear topological spaces which are not complete, and hence are not Pták spaces.

From open mapping theorems arise restrictions on continuous images of certain types of spaces. As an application of his open mapping theorem, Banach ([2], p. 38), showed that the continuous linear image of a complete metric linear space in a space of the same 
kind is either meager or the whole space. Robertson and Robertson [9] extend this result in locally convex linear topological spaces by use of fully complete or Pták spaces. Note that a linear subspace of a locally convex linear topological space which is not both dense and barrelled is a meager subspace. Moreover, Pták spaces include Fréchet spaces (complete metric linear spaces which are locally convex). Thus the following modification of the Robertson and Robertson result includes Banach's result restricted to Fréchet spaces, but with a more precise requirement than meagerness: If $g$ is a continuous linear mapping of a Pták space $E$ into a Hausdorff locally convex linear topological space $F$, then $g$ maps $E$ onto $F$, if $g[E]$ is both dense in $F$ and barrelled.

Recently, Saxon [11] has shown that each infinite dimensional Banach space contains a dense barrelled subspace which is not Baire, and so, as each must be proper, the above result implies that none is a continuous linear image of a Pták space. This is also true for pseudo-complete locally convex linear topological space in place of Pták space. However, this result is not based on an open mapping theorem, but rather on the difference theorem via Theorem 3.1; the proof of this theorem is the main subject of $\S 3$ which also contains an extension of a result of Aarts and Lutzer. Section 4 discusses the conditions of Theorem 3.1 and states some corollaries to it, while $\S 2$ gives some definitions and earlier results, and $\S 5$ contains examples relating to Theorem 3.1.

2. Definitions and remarks. A topological space is quasi-regular if and only if each nonempty open set contains the closure of a nonempty open set. A family $\mathscr{B}$ of nonempty open sets is a pseudobase if and only if each nonempty open set contains an element of $\mathscr{B}$. Finally, a quasi-regular topological space is pseudo-complete if and only if there is a sequence $\left(\mathscr{B}_{n}\right)$ of pseudo-bases such that if the terms of the sequence $\left(U_{n}\right)$ are elements of terms of $\left(\mathscr{B}_{n}\right)$, respectively, and each $U_{n}$ contains the closure of $U_{n+1}$, then the intersection $\bigcap_{n} U_{n}$ is nonempty.

A subset of a topological space is rare if its closure has no interior, and is meager if it may be covered by a countable family of rare sets. A topological space is a Baire space if and only if each nonempty open set is not meager. The closure of a Baire subspace of a topological space is a Baire space. In case $E$ is a linear topological space, $E$ is a Baire space if and only if $E$ is not meager. Oxtoby [8] shows that each pseudo-complete space is a Baire space.

Each topologically complete metrizable space is pseudo-complete. A linear topological space is regular, and so is quasi-regular. Todd [13] shows that a linear metrizable space is pseudo-complete if and 
only if it is complete; however, a complete linear topological space need not be pseudo-complete.

A mapping $f$ from a topological space $X$ to a topological space $Y$ is almost open if and only if $(f N)^{-} \cap(f X)$ is a neighborhood of $f(u)$ in the subspace $(f X)$ of $Y$, whenever $N$ is a neighborhood of $u$ in $X$. An open mapping is defined in the same manner, but without the closure used. The image of a Baire space under an almost open continuous mapping is a Baire space. In case $E$ and $F$ are linear topological spaces and $g$ is a linear mapping of $E$ onto $F$, the mapping $g$ is almost open if and only if $(g U)^{-}$is a neighborhood of 0 in $F$ whenever $U$ is a neighborhood of 0 in $E$.

A topological space $X$ has a continuous metric if and only if there is a metric on the set $X$ which induces a topology on $X$ weaker than the topology of $X$. It is easy to see that a topological space $X$ has a continuous metric $d$ if $d$ is a metric on $X$ and is continuous as a function from $X \times X$ is the real numbers. Todd [13] shows that a linear topological space $E$ has a continuous translation invariant metric if and only if $E$ has a countable family of open sets which intersects in a singleton.

For other terms, the paper generally follows Horváth [4] and Kelley, Namioka, et al. [6], for example, $A^{-i}$ is the interior of the closure of set $A$; however, locally convex linear topological space shortens to convex space and the name of a topological property follows "convex" and "linear."

3. Images of a linear pseudo-complete spaces. The main theorem follows.

THEOREM 3.1. The image of a linear pseudo-complete space under a linear continuous almost open mapping is complete, if its completion has a continuous metric.

Proof. Let $E$ be a linear topological space which is pseudo-complete, $g$ be a linear continuous almost open mapping from $E$ to a linear topological space $F$. We may suppose that $F$ is the completion of $g E$ and let $d$ be a continuous metric on $F$. Finally, suppose $\left(\mathscr{B}_{n}\right)$ is a sequence of pseudo-bases for $E$ as in the definition of pseudocompleteness.

Claim. There is a sequence $\left(\mathscr{C}_{n}\right)$ with each $\mathscr{C}_{n}$ a subfamily of $\mathscr{B}_{n}$ such that, with $\mathscr{C}_{0}=\{E\}$, for each $k \geqq 0$,

(0) $U_{k}=\bigcup\left\{(g C)^{-i}: C \in \mathscr{C}_{k}\right\}$ is dense in $F$,

(1) $\left\{(g C)^{-i}: C \in \mathscr{C}_{k}\right\}$ is a disjoint family,

for each $C$ in $\mathscr{C}_{k+1}$, 
(2) $d$-diam $(g C)<1 /(k+1)$, and

(3) some $D$ in $\mathscr{C}_{k}$ contains $C^{-}$.

Proof of claim. Clearly $\mathscr{C}_{0}$ satisfies (0) and (1). Suppose $m \geqq$ -1 , and $\mathscr{C}_{0}, \cdots, \mathscr{C}_{m+1}$ satisfy $(0)$ and (1) for $0 \leqq k \leqq m+1$ and satisfy (2) and (3) for $0 \leqq k \leqq m$.

Let $n=m+1$ and $\mathscr{C}$ be the family of all elements $C$ of $\mathscr{B}_{n+1}$ which satisfy (2) and (3) for $k=n$. Let $\mathscr{C}_{n+1}$ be maximal among subfamilies $\mathscr{D}$ of $\mathscr{C}$ satisfying (1) for $\mathscr{C}_{k}$ replaced by $\mathscr{D}$.

Assume $U_{n+1}=\bigcup\left\{(g C)^{-i}: C \in \mathscr{C}_{n+1}\right\}$ is not dense in $F$. Since $U_{n}$ is dense, there is an element $D$ of $\mathscr{C}_{n}$ such that $(g D)^{-i} \backslash U_{n+1}^{-}$is nonempty. Let $V$ be a nonempty open subset of $F$ with $d$-diam $(V)<$ $1 /(n+1)$ and $V^{-}$contained in $(g D)^{-i} \backslash U_{n+1}^{-}$. As $V$ meets $g D$, there is $B$ in $\mathscr{B}_{n+1}$ with $B^{-}$contained in $D \cap g^{-1} V$.

Now $g B \cap V^{-} \subset(g D)^{-i} \backslash U_{n+1}^{-}$, thus $d$-diam $(g B)<1 /(n+1)$, and, as $B^{-} \subset D \in \mathscr{C}_{n}, B$ is an element of $\mathscr{C}$. Moreover, $(g B)^{-i}$ is in the complement of $U_{n+1}$, so $\mathscr{D}=\mathscr{C}_{n+1} \cup\{B\}$ is a subfamily of $\mathscr{C}$ satisfying (1) for $\mathscr{C}_{k}$ replaced by $\mathscr{D}$. From the maximality of $\mathscr{C}_{n+1}, B$ is in $\mathscr{C}_{n+1}$. Hence $(g B)^{-i} \subset U_{n+1}$, a contradiction as $g$ is almost open, and $B$ is a nonempty open set.

Because $g$ is almost open and continuous and $E$ is a Baire space, the completion $F$ of $g E$ is itself a Baire space. By (0) each $U_{n}$ is dense and open in the Baire space $F$, so $A=\bigcap_{n} U_{n}$ is a dense $G_{\delta}$ subset of $F$, and thus, necessarily, nonmeager in $F$. By the difference theorem (see Kelley-Namioda [6], 10.4, p. 92), the difference set $(A-A)$ is a neighborhood of 0 in $F$.

We now show each $y$ in $A$ is in $g E$, and hence $g E$ equals $F$, which will complete the proof. There are $C_{n} \in \mathscr{C}_{n}$ with $y$ in each $\left(g C_{n}\right)^{-i}$. From (2), $\bigcap_{n}\left(g C_{n}\right)^{-i}=\{y\}$. For $n$ fixed, there is $D$ in $\mathscr{C}_{n}$ containing $C_{n+1}^{-}$by (3). Now, we have

$$
y \in\left(g C_{n}\right)^{-i} \cap\left(g C_{n+1}\right)^{-i} \subset\left(g C_{n}\right)^{-i} \cap(g D)^{-i},
$$

so $C_{n}=D$ by (1), and $C_{n}$ contains $C_{n+1}^{-}$for each $n$. From pseudocompleteness there is an $x$ in $\bigcap_{n} C_{n}$, and so, we have, since $g$ is almost open,

$$
g(x) \in \bigcap_{n}\left(g C_{n}\right) \subset \bigcap_{n}\left(g C_{n}\right)^{-i}=\{y\} .
$$

Therefore $y$ is in $g E$.

This result generalizes the following result arising from [13]; the proofs are similar.

COROLLARY 3.2. If the topology of the completion of a linear 
topological space $E$ contains a countable family which intersects in a singleton, then $E$ is complete if it is pseudo-complete.

Proof. Existence of such a countable family is equivalent to the existence of a continuous metric on the completion of $E$. To apply the theorem, use the injection mapping of $E$ into itself.

The claim in the proof of the theorem does not depend on linearity and may be used in proving the following extension of a theorem of Aarts and Lutzer [1], where now almost open replaces open.

TheOREM 3.3 (Aarts and Lutzer). Suppose $X$ is pseudo-complete and $f: X \rightarrow Y$ is continuous, onto and almost open. If $Y$ is metrizable, then $Y$ contains a dense subspace which has a complete metric and is zero-dimensional. In particular, $Y$ is pseudo-complete.

4. The conditions. We now consider contexts in which almost open mappings and continuous metrics arise.

A property of barrelled spaces $F$ (see Horvath [4], Prop. 1, p. 296 ) is that each linear surjection of a convex space $E$ onto $F$ is almost open; in fact, this property characterizes barrelled spaces. A similar statement holds for ultra-barrelled spaces (see W. Robertson [10]) in place of barrelled spaces and linear topological space in place of convex space. Each linear Baire space is ultra-barrelled, and a convex space which is ultra-barrelled is barrelled. Each of these implications is strict ([10]). Interest in linear Baire spaces is further enhanced by the following characterization of Saxon [12]: A linear topological space is a Baire space if and only if no absorbing balanced set is rare.

The following two corollaries of Theorem 3.1 follow without difficulty from the above discussion.

CoRollary 4.1. The image of a convex pseudo-complete space under a linear continuous mapping is complete, if it is barrelled and its completion has a continuous metric.

COROLLARY 4.2. The image of a linear pseudo-complete space under a linear continuous mapping is complete, if it is an ultrabarrelled space and its completion has a continuous metric.

Since a complete linear metrizable space is pseudo-complete, the following gives the result of Banach discussed in the introduction, but without use of the open mapping theorem.

COROLlary 4.3. The linear continuous image of a linear pseudo- 
complete space in a linear metrizable space is either meager or the whole space.

Proof. Suppose $g: E \rightarrow F$ are as indicated. If $g E$ is not meager in $F$, then it is dense in $F$. Moreover, $g E$ is not meager in itself, hence $g E$ is a Baire space, and so is ultra-barrelled. Therefore $g$ is almost open. As $F$ is metrizable, its completion is metrizable, and so the completion of $g E$ has a continuous metric. Hence $g E$ is complete and, in particular, closed in $F$. Thus $g E$ is $F$.

As may be seen, meager may be replaced in this corollary by not both dense and ultra-barrelled or, in case the domain space is convex, by not both dense and barrelled. Thus, for example, no proper linear subspace of a Banach space which is both dense and barrelled is the linear continuous image of a convex pseudo-complete space.

Now let us consider contexts in which a linear topological space has a continuous metric. Of course such a metric exists if the linear topological space has a continuous semi-norm or continuous para-norm which is 0 only at the zero of the space. However, the following is more specific.

THEOREM 4.4. If a linear topological space $E$ has a countable total family of linear continuous functionals, then $E$ has a continuous metric.

Proof. Suppose $\left(f_{n}\right)$ is a sequence of linear continuous functionals on $E$ which is total, i.e. if $f_{n} x=0$ for all $n$, then $x=0$. Now $U_{m n}=$ $\left\{x \in E:\left|f_{n} x\right|<1 / m\right\}$ gives a countable family of open sets whose intersection $\bigcap_{m n} U_{m n}$ is a singleton. Therefore $E$ has a continuous metric.

(A more direct proof comes from consideration of the Fréchet combination $d(x, y)=\sum_{n \geqq 1} 2^{-n}\left|f_{n}(x-y)\right| /\left(1+\left|f_{n}(x-y)\right|\right)$.)

Thus, for example, a linear Hausdorff space with a Schauder basis (see McArthur [7]) has a continuous metric. Also, for $E$ a linear topological space with continuous dual $E^{\prime}$, if $E^{\prime}$ contains a countable total family, then any linear topology on $E$ with the same dual $E^{\prime}$ has a continuous metric; in particular, the weak topology on $E$ has a continuous metric. Clearly, the countable total family of continuous functionals in Theorem 4.4 may be replaced by a sequence $\left(p_{n}\right)$ of continuous para-norms for which $p_{n}(x)=0$, for all $n$, implies $x=0$.

Even a continuous norm on a normed linear space need not extend 
to a continuous norm on the completion of the space, as may be seen in Example 5.8. However, a translation invariant continuous pseudometric on a linear topological space extends uniquely to a continuous pseudo-metric on the completion of the space. The result is essentially a simple observation of uniform spaces, but we shall state it here for linear topological spaces.

THeOREM 4.5. If linear topological space $E$ has a translation invariant continuous pseudo-metric d', then $d^{\prime}$ has a unique extension $d$ to the completion $E^{\wedge}$ of $E$ which is a translation invariant continuous pseudo-metric on $E^{\wedge}$.

Proof. Clearly $d^{\prime}: E \times E \rightarrow R$ is uniformly continuous. From Kelley ([5], Th. 26, p. 195), $d^{\prime}$ has a unique continuous extension $d$ to $E^{\wedge} \times E^{\wedge}$. It may be verified that $d$ is a translation invariant pseudo-metric on $E^{\wedge}$, and so a continuous pseudo-metric on $E^{\wedge}$.

Of interest are conditions under which a translation invariant continuous metric which induces a linear topology, may be extended to a continuous metric on the completion of a space. These are essentially characterized by a result of W. Robertson [10], restated here in terms of nets: Suppose that $E$ is a linear Hausdorff space under each of two topologies $\xi$ and $\eta$, with $\xi$ finer than $\eta$, and that $i$ is the identity mapping $i:(E, \xi) \subseteq(E, \eta)$. Then the extension $i^{\wedge}$ of $i$ is a one-to-one mapping of $(E, \xi)^{\wedge}$ into $(E, \eta)^{\wedge}$ if and only if each Cauchy net in $(E, \xi)$ which converges in $(E, \eta)$ also converges in $(E, \xi)$.

With this, we obtain the following.

THEOREM 4.6. If $d$ is a translation invariant continuous metric on a linear topological space $E$ which induces a linear topology on $E$, then $d$ has an extension to a continuous metric on the completion $E^{\wedge}$ of $E$ if and only if each Cauchy net in $E$ which converges in $(E, d)$ also converges in $E$.

Proof. In the W. Robertson theorem, let $\xi$ be the topology of $E$ and $\eta$ be the linear metric topology induced on $E$ by $d$. With $i: E \subseteq(E, d)$, the extension $i^{\wedge}: E^{\wedge} \rightarrow(E, d)^{\wedge}$ of $i$ is one-to-one if and only if each Cauchy net in $E$ which converges in $(E, d)$ is convergent in $E$.

Suppose the extension $i^{\wedge}$ is one-to-one. In any case, $(E, d)^{\wedge}$ is a linear metrizable space and the extension $d^{\wedge}$ of $d$ from $(E, d)$ to $(E, d)^{\wedge}$ is a translation invariant metric inducing the topology of $(E, d)^{\wedge}$. As $i^{\wedge}$ is continuous and assumed one-to-one, $d^{-}(x, y)=d\left(i^{\wedge} x, i^{\wedge} y\right)$ is 
a continuous metric on $E^{\wedge}$, which is an extension of $d$ on $E$ to $E^{\wedge}$.

Now suppose $d$ has an extension to a continuous metric $d^{-}$on $E^{\wedge}$, and let $\left(x_{\alpha}\right)$ be a Cauchy net in $E$ which converges to $x$ in $(E, d)$. The net $\left(x_{\alpha}\right)$ converges to some $y$ in $E^{\wedge}$, and so

$$
d^{-}(x, y)=\lim _{\alpha} d^{-}\left(x, x_{\alpha}\right)=\lim _{\alpha} d\left(x, x_{\alpha}\right)=0 .
$$

Thus $x=y$, and $\left(x_{\alpha}\right)$ converges in $E$.

5. Examples and an open question. In [8], Oxtoby showed that the Cartesian product of any family of pseudo-complete spaces is pseudo-complete. In this section, we shall see that any product of an uncountable family of nontrivial pseudocomplete spaces contain proper dense subspaces which are pseudocomplete. Other examples show the necessity of the almost open requirement of Theorem 3.1 and the difficulty of obtaining it even with all other properties of Theorem 3.1 satisfied. The final example shows that a continuous norm need not have a continuous extension to a norm. The paper ends with an open question.

For the first objective, we shall need several technical propositions.

Proposition 5.1. For each sequence $\left(\mathscr{C}_{n}\right)$ of pseudo-bases of a quasi-regular space $X$, there is a sequence $\left(\mathscr{B}_{n}\right)$ of pseudo-bases with each $\mathscr{B}_{n}$ a subfamily of $\mathscr{C}_{n}$ and the family of closure of elements of $\mathscr{B}_{n+1}$ a refinement of $\mathscr{B}_{n}$.

Proof. We define $\left(\mathscr{B}_{n}\right)$ by induction. Let $\mathscr{B}_{1}$ equal $\mathscr{C}_{1}$, and suppose we have $\mathscr{B}_{1}, \cdots, \mathscr{B}_{n}$ satisfying the requirements. Let $\mathscr{B}_{n+1}=$ $\left\{C \in \mathscr{C}_{n+1}\right.$ : some $H$ in $\mathscr{B}_{n}$ contains $\left.C^{-}\right\}$. Let $U$ be a nonempty open subset of $X$. Now $U$ contains some element $B$ of $\mathscr{B}_{n}$, and, as $X$ is quasi-regular, $B$ contains the closure of some nonempty open set $V$. $\mathscr{C}_{n+1}$ is a pseudo-base, so some element $C$ of $\mathscr{C}_{n+1}$ is contained in $V$. Now $U \supset B \supset V^{-} \supset V \supset C$, hence $U$ contains $C$ and $B$ contains $C^{-}$, thus $C$ is in $\mathscr{B}_{n+1}$. Therefore $\mathscr{B}_{n+1}$ is a pseudo-base for $X$, and the family of closures of elements of $\mathscr{B}_{n+1}$ refines $\mathscr{B}_{n}$.

Proposition 5.2. If $X$ is pseudo-complete and nonempty, then there is a sequence $\left(\mathscr{B}_{n}\right)$ of pseudo-bases of $X$ as in the definition of pseudo-completeness and such that each $\mathscr{B}_{n}$ contains $X$.

Proof. Let $\left(\mathscr{C}_{n}\right)$ be a sequence of pseudo-bases for $X$ which satisfies the requirements of pseudo-completeness for $X$. Suppose $\left(\mathscr{B}_{n}^{\prime}\right)$ is a sequence of pseudo-bases related to $\left(\mathscr{C}_{n}\right)$ as in Proposition 5.1, and let $\mathscr{B}_{n}=\mathscr{B}_{n}^{\prime} \cup\{X\}$. We need only see, for any sequence $\left(U_{n}\right)$ 
of elements of the terms of $\left(\mathscr{B}_{n}\right)$ respectively, with each $U_{n}$ containing $U_{n+1}^{-}$, that the intersection $\bigcap_{n} U_{n}$ is nonempty. Should each $U_{n}$ be $X$, then $\bigcap_{n} U_{n}=X \neq \varnothing$. Otherwise, for some $m, U_{m} \neq X$. As each $U_{n}$ contains $U_{n+1}$, each $U_{k} \neq X$ for $k \geqq m$, and we may suppose $m>2$. Now each $U_{k}$ is in $\mathscr{B}_{k}^{\prime}$ for $k \geqq m$. As the family of closures of elements of each $\mathscr{B}_{n+1}^{\prime}$ refines $\mathscr{B}_{n}^{\prime}$, there is an element $V_{m-1}$ of $\mathscr{B}_{m-1}^{\prime}$ with $V_{m-1}$ containing $U_{m}^{-}$, and, by induction, elements $V_{k}$ of $\mathscr{B}_{k}^{\prime}$ for $k<m-1$, so that $V_{k}$ contains $V_{k+1}^{-}$. Finally $\left(V_{n}\right)$ with $V_{n}=$ $U_{n}$, for $n \geqq m$, satisfies $V_{n} \in \mathscr{\mathscr { B }}_{n}^{\prime}$ and $V_{n} \supset V_{n+1}^{-}$so that $\bigcap_{n} U_{n}=$ $\bigcap_{n \geqq m} U_{n}$ contains $\left(\bigcap_{k<m} V_{k}\right) \cap\left(\bigcap_{k \geqq m} U_{k}\right)=\bigcap_{n} V_{n} \neq \varnothing$.

The following theorem and, especially, its corollary may be compared with similar facts concerning barrelled, linear Baire and other closely related spaces in Corollary 4.12 of [14].

THEOREM 5.3. Let $X$ be a product of a family $\left\{X_{j}: j \in J\right\}$ of pseudo-complete spaces, and $x_{0}$ be in $X$. If $X_{0}$ is a subspace of $X$ containing all $x$ with $x(j)=x_{0}(j)$ for all but a countable subset of $J$, then $X_{0}$ is pseudo-complete.

Proof. For each $j$ in $J$, let $\left(\mathscr{B}_{n}(j)\right)$ be a sequence of pseudobases for $X_{j}$ as in the definition of pseudo-completeness for $X_{j}$. We may suppose each $\mathscr{B}_{n}(j)$ contains $X_{j}$. Let each $\mathscr{C}_{n}$ consist of products of $B_{j} \in \mathscr{B}_{n}(j)$ where all but a finite number of $B_{j}$ are $X_{j}$, and take $\mathscr{B}_{n}$ equal to $\left\{C \cap X_{0}: C \in \mathscr{C}_{n}\right\}$.

Clearly each $\mathscr{B}_{n}$ is a pseudo-base for the dense subspace $X_{0}$ of $X$. Moreover, $X_{0}$ is quasi-regular. Now suppose $U_{n} \in \mathscr{B}_{n}$ and $U_{n}$ contains $U_{n+1}^{-} \cap X_{0}$ for each $n$. There are $B_{n}^{j} \in \mathscr{B}_{n}(j)$ with $U_{n}=$ $\left(\Pi_{J} B_{n}^{j}\right) \cap X_{0}$, and so, for each fixed $n$,

$$
\begin{aligned}
\left(\Pi_{J} B_{n}^{j}\right) \cap X_{0} & =U_{n} \supset U_{n+1}^{-} \cap X_{0}=\left[\left(\Pi_{J} B_{n+1}^{j}\right) \cap X_{0}\right]^{-} \cap X_{0} \\
& =\left(\Pi_{J} B_{n+1}^{j}\right)^{-} \cap X_{0}=\left(\Pi_{J} B_{n+1}^{j-1}\right) \cap X_{0} .
\end{aligned}
$$

Let $K$ be a countable subset of $J$ such that for each $j$ in $J \backslash K, B_{n}^{j}$ is $X_{j}$ for all $n$. For any $u$ in $B_{n+1}^{h-}$, define $x$ in $X_{0}$ by $x(h)=u$, choose any $x(j)$ in $B_{n+1}^{j}$ for $j$ in $K \backslash\{h\}$, and $x(j)=x_{0}(j)$ otherwise. Thus $x$ is in $U_{n}$, and so $u=x(h)$ is in $B_{n}^{h}$. Hence $B_{n}^{j}$ contains $B_{n+1}^{j-1}$ for all $n$ and $j$. Now $\bigcap_{n} B_{n}^{j}$ is nonempty for each $j$. Therefore there is an $x$ in $X$ such that $x(j)$ is in $\bigcap_{n} B_{n}^{j}$ for all $j$, and, additionally, $x(j)=$ $x_{0}(j)$ for $j$ in $J \backslash K$. Thus $x$ is in $X_{0}$. Finally, $x$ is in the intersection $\bigcap_{n} U_{n}$ so that $\left(\mathscr{B}_{n}\right)$ satisfies the requirements of pseudo-completeness for $X_{0}$.

The following is a direct consequence of this theorem. 
CoRollary 5.4. Let $E$ be a product of a family of linear pseudo-complete spaces. If $E_{0}$ is a linear subspace of $E$ which contains all elements of $E$ whose coordinates are zero except for a countable subset, then $E_{0}$ is a linear pseudo-complete space.

An example of [13] is a convex space which has a continuous norm and which is complete yet not pseudo-complete. The existence of linear pseudo-complete spaces which are not complete was left open. The following exhibits such spaces.

EXAMPLE 5.5. Suppose $E$ is an uncountable product of nontrivial linear complete metric spaces, and let $E_{0}$ be the linear subspace of $E$ consisting of all elements $x$ of $E$ whose coordinates are zero except for a countable subset. The linear topological space $E_{0}$ is pseudocomplete by the corollary above; however, it is a dense proper subspace of $E$, and so not a complete linear topological space. Thus pseudo-completeness does not imply completeness.

Suppose the factor spaces in the above example are all convex, then, as Pták spaces are complete, we have examples of convex pseudo-complete spaces which are not Pták spaces.

The following example suggests the importance of the almost open requirement in the main theorem.

EXAMPLE 5.6. Let $g$ be the injection of $E=\left(\iota_{1},\|\|_{1}\right)$ into $F=$ $\left(\ell_{1},\|\|_{\infty}\right) . \quad E$ is a Banach space, and so is pseudo-complete; $F$ is a normed space, and so its completion has a continuous metric; finally, $g$ is a linear continuous mapping which is both one-to-one and onto. However, the image $g E=F$ is not complete. To see, independently of Theorem 3.1, that $g$ is not almost open, observe that $E$ is a Pták space, and so each linear continuous mapping of $E$ into a convex Hausdorff space is open if it is almost open.

Even with what appear to be strong conditions, in particular, all the conditions, except for almost open, of the hypothesis and conclusion of Theorem 3.1, a mapping need not be almost open. This is born out in the following.

ExAmPLe 5.7. Let $E=(E,\|\|)$ be an infinite dimensional Banach space for which there is a sequence of linear continuous functionals which is total. Let $F=(E, \sigma)$ where $\sigma$ is the convex topology for $E$ with local base consisting of all polars $\left(f_{n}\right)^{0}=\left\{x \in E:\left|f_{n} x\right| \leqq 1\right.$, $n \in \omega\}$ of sequences $\left(f_{n}\right) \subset E^{\prime}$ which norm converge to 0 in $E^{\prime}$. This is the weak-bounded topology or bw-topology for $E$ (Day [3], Def. 2, 
p. 41). It may be seen that $\sigma$ is weaker than the norm topology and stronger than the weak topology for $E$. Thus, the injection $g$ of pseudo-complete space $E$ into $F$ is linear, one-to-one, onto and continuous. Now $F$ is complete or not according as $E$ is or is not reflexive ([3], Th. 2, p. 57). When $E$ is reflexive, the completion of $F$ clearly has a continuous metric. However, $g$ is never almost open. For let $S$ be the closed unit ball of $E$, then $S=g S$ is closed in $F$ since it is closed in the weak topology for $E$. Suppose $\left(f_{n}\right)^{0}$ is a neighborhood of 0 in $F$, as above, and choose $N$ such that $\left\|f_{n}\right\| \leqq$ $1 / 2$ for $n \geqq N$, and $x$ in $E$ such that $\|x\|=2$ and $f_{n} x=0$ for $1 \leqq$ $n \leqq N$. Thus $x$ is in $\left(f_{n}\right)^{0}$, yet not in $S$, and so $S$ is not a neighborhood of 0 in $F$.

The following example shows that a translation invariant continuous metric on a linear topological space need not have a continuous extension which is a metric on the completion.

EXAMPLE 5.8. Let $E$ be an infinite dimensional Banach space under the norm $p$, and suppose \|\| is a norm on $E$ which induces a linear topology strictly finer than that induced by $p$, so that $p$ is a continuous norm on $(E,\|\|)$. Now $(E,\|\|)$ is not complete, hence there is a Cauchy sequence $\left(x_{n}\right)$ in $(E,\|\|)$ which does not converge. Suppose it converges to $y$ in $(E,\|\|)^{\wedge}$. It also converges to some $x$ in $(E, p)$. Let $p^{-}$be the continuous extension of $p$ to $(E,\|\|)^{\wedge}$. Clearly, $p^{-}(y-x)=\lim _{n} p^{-}\left(x_{n}-x\right)=\lim _{n} p\left(x_{n}-x\right)=0$, yet $y-x \neq$ 0 , so that $p^{-}$is not a norm on $(E,\|\|)^{\wedge}$. Now $p(u-v)$ gives a translation invariant continuous metric on $(E,\|\|)$; its extension to $(E,\|\|)^{\wedge}$ is $p^{-}(u-v)$, which is not a metric.

Open question. Since there are convex pseudo-complete spaces which are not Pták spaces, there is a linear continuous mapping $g$ of such a space $E$ onto a convex Hausdorff space $F$ such that $g$ is almost open, but not open. Of more interest in the context of Theorem 3.1 would be for $F$, in addition, to be metrizable or for its completion to have a continuous metric. Such an example remains to be found.

\section{REFERENCES}

1. J. M. Aarts and D. J. Lutzer, Pseudo-completeness and the product of Baire spaces, Pacific J. Math., 48 (1973), 1-10.

2. S. Banach, Théorie des Operations Linéares, Monografie Mat., PWN, Warsaw, 1932.

3. M. M. Day, Normed Linear Spaces, Ergebnisse der Mathematik und ihrer Grenzebiete, Neue Folge. Heft 21, Springer, Berlin, 1958.

4. J. Horváth, Topological Vector Spaces and Distributions, Vol. 1, Addison-Wesley, Reading, Mass. 1966. 
5. J. L. Kelley, General Topology, D. Van Nostrand, Princeton, New Jersey, 1955.

6. - I. Namioka et al., Linear Topological Spaces, D. Van Nostrand, Princeton, New Jersey, 1963.

7. C. W. McArthur, Developments in Schauder basis theory, Bull. Amer. Math. Soc., 78 (1972), 877-908.

8. J. C. Oxtoby, Cartesian products of Baire spaces, Fund. Math., 49 (1961), 157-166. 9. A. P. Robertson and W. J. Robertson, On the closed graph theorem, Proc. Glasgow Math. Assoc., 3 (1956), 9-12.

10. W. Robertson, Completions of topological vector spaces, Proc. London Math. Soc., (3) 8 (1958), 242-257.

11. S. A. Saxon, Some normed barrelled spaces which are not Baire, Math. Ann., 209 (1974), 153-160.

12. , Two characterizations of linear Baire spaces, Proc. Amer. Math. Soc., 45 (1974), 204-208.

13. A. R. Todd, Pseudo-completeness in linear metrizable spaces, (to appear).

14. and S. A. Saxon, A property of locally convex Baire spaces, Math. Ann., 206 (1973), 23-34.

Received March 7, 1975 and in revised form November 4, 1975. Some of the observations of this paper are included in the author's dissertation written at the University of Florida (1972) under the supervision of Dr. Stephen A. Saxon.

Brooklyn College of the City University 


\section{PACIFIC JOURNAL OF MATHEMATICS}

EDITORS

RICHARD ARENS (Managing Editor)

University of California

Los Angeles, California 90024

R. A. BEAUMONT

University of Washington

Seattle, Washington 98105
J. DugundJI

Department of Mathematics

University of Southern California

Los Angeles, California 90007

D. Gilbarg and J. Milgram

Stanford University

Stanford, California 94305

\section{ASSOCIATE EDITORS}

E. F. BECKENBACH

B. H. NeumanN

F. WOLF

K. YosHIDA

\section{SUPPORTING INSTITUTIONS}

UNIVERSITY OF BRITISH COLUMBIA

UNIVERSITY OF SOUTHERN CALIFORNIA

CALIFORNIA INSTITUTE OF TECHNOLOGY

UNIVERSITY OF CALIFORNIA

STANFORD UNIVERSITY

UNIVERSITY OF TOKYO

MONTANA STATE UNIVERSITY

UNIVERSITY OF UTAH

UNIVERSITY OF NEVADA

WASHINGTON STATE UNIVERSITY

NEW MEXICO STATE UNIVERSITY

UNIVERSITY OF WASHINGTON

OREGON STATE UNIVERSITY

UNIVERSITY OF OREGON

OSAKA UNIVERSITY

AMERICAN MATHEMATICAL SOCIETY
NAVAL WEAPONS CENTER

Printed in Japan by International Academic Printing Co., Ltd., Tokyo, Japan 


\section{Pacific Journal of Mathematics}

\section{Vol. 63, No. $1 \quad$ March, 1976}

Ralph Artino, Gevrey classes and hypoelliptic boundary value problems ....... 1

B. Aupetit, Caractérisation spectrale des algèbres de Banach commutatives .... 23

Leon Bernstein, Fundamental units and cycles in the period of real quadratic

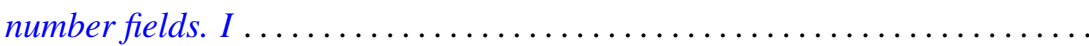

Leon Bernstein, Fundamental units and cycles in the period of real quadratic number fields. II.................................... 63

Robert F. Brown, Fixed points of automorphisms of compact Lie groups ........

Thomas Ashland Chapman, Concordances of noncompact Hilbert cube

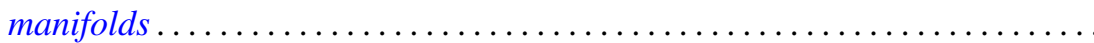

William C. Connett, V and Alan Schwartz, Weak type multipliers for Hankel

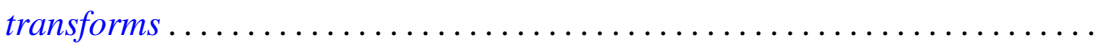

John Wayne Davenport, Multipliers on a Banach algebra with a bounded approximate identity .....................................

Gustave Adam Efroymson, Substitution in Nash functions ................ 137

John Sollion Hsia, Representations by spinor genera ..................

William George Kitto and Daniel Eliot Wulbert, Korovkin approximations in

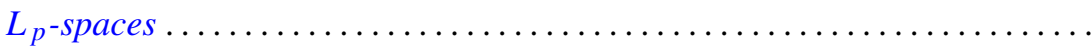

Eric P. Kronstadt, Interpolating sequences for functions satisfying a Lipschitz. condition ...........................................

Gary Douglas Jones and Samuel Murray Rankin, III, Oscillation properties of certain self-adjoint differential equations of the fourth order...

Takaŝi Kusano and Hiroshi Onose, Nonoscillation theorems for differential

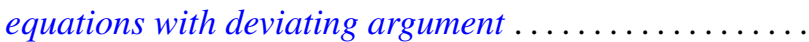

David C. Lantz, Preservation of local properties and chain conditions in commutative group rings. ...

Charles W. Neville, Banach spaces with a restricted Hahn-Banach extension property....

Norman Oler, Spaces of discrete subsets of a locally compact group ...

Robert Olin, Functional relationships between a subnormal operator and its minimal normal extension.

Thomas Thornton Read, Bounds and quantitative comparison theorems for nonoscillatory second order differential equations ...... .

Robert Horace Redfield, Archimedean and basic elements in completely distributive lattice-ordered groups...

Jeffery William Sanders, Weighted Sidon sets

Aaron R. Todd, Continuous linear images of pseudo-complete linear topological spaces.

J. Jerry Uhl, Jr., Norm attaining operators on $L^{1}[0,1]$ and the Radon-Nikodým property.

William Jennings Wickless, Abelian groups in which every endomorphism is a left multiplication. 